\title{
INCOMPRESSIBLE SURFACES IN KNOT SPACES
}

\author{
BY \\ HERBERT C. LYON( $\left.{ }^{1}\right)$
}

\begin{abstract}
The following theorems are proved.
THEOREM 1. There exist infinitely many distinct, prime, Neuwirth knots, each of which has the property that its complement contains closed, incompressible surfaces of arbitrarily high genus.
\end{abstract}

THEOREM 2. There exists a genus one knot which has incompressible spanning surfaces of arbitrarily high genus.

1. Introduction. We know from the work of Waldhausen, [8], [9], [10], that the only closed, incompressible surfaces embedded in the complement of a torus knot are boundary parallel tori. It has been conjectured that this result generalizes to all prime Neuwirth knots. Our first theorem shows this conjecture to be false; in fact, there is not even an upper bound on the genera of the closed, incompressible surfaces embedded in the complements of some particular prime Neuwirth knots.

The proof of our second theorem uses the same basic construction to find a genus one knot which has incompressible spanning surfaces of arbitrarily high genus. These results are somewhat similar to those obtained by Alford in [1] and Schaufele in [7]. They are also related to Questions $\mathrm{L}$ and $\mathrm{T}$ as asked by Neuwirth in [6].

I wish to acknowledge the generous assistance that William Jaco has given me in preparing this paper.

2. Preliminaries. Our notation generally parallels that of Haken [4] and Waldhausen [8], [9], [10], [11]. All work is done in the piecewise linear category. The $n$-sphere is denoted by $S^{n}$ for $n=1,2$, or 3 . The unit interval $[0,1]$ is symbolized by $I$, and $D^{2}$ denotes the 2-cell.

A manifold is a compact, connected orientable 3-manifold, with or without boundary. If $M$ is a manifold and $S$ is a surface, we denote the boundary of $M$ and the boundary of $S$ by Bd $M$ and Bd $S$, respectively. When Bd $M=\varnothing, M$ is closed; and when Bd $S=\varnothing, S$ is closed. A surface $S$ in a manifold $M$ is, in general, properly embedded, i.e. $S \cap \operatorname{Bd} M=\operatorname{Bd} S$. An arc $\alpha$ in a surface $S$ is a spanning arc if $\alpha \cap \operatorname{Bd} S=\operatorname{Bd} \alpha$

Received by the editors July 30, 1970.

AMS 1970 subject classifications. Primary 55A25; Secondary 55A35, 55A40.

Key words and phrases. Closed surface, genus, incompressible surface, knot, knot space, Neuwirth knot, prime knot, spanning surface.

${ }^{(1)}$ The results in this paper are contained in the author's doctoral dissertation, written at the University of Michigan under the direction of William Jaco and supported by a Danforth Graduate Fellowship. 
The closure of an object $(\cdots)$ is denoted by $\mathrm{Cl}(\cdots)$. Its interior is Int $(\cdots)$, and its Euler characteristic is $\chi(\cdots)$. A regular neighborhood of a subcomplex $(\cdots)$ in a manifold $M$ is symbolized by $N(\cdots)$. The notation $N(\cdots)$ is also used for regular neighborhoods of subcomplexes in surfaces. However, the context will make it clear as to which notion we are using.

A product neighborhood $I(S)$ of a surface $S$ in a manifold $M$ is an embedding of $S \times I$ as a subcomplex of $M$ so that $S=S \times\left\{\frac{1}{2}\right\}$. If $S$ is properly embedded in $M$, then we require that $(S \times I) \cap(\mathrm{Bd} M)=(\mathrm{Bd} S) \times I$. In this case $I(S)$ is a regular neighborhood of $S$.

Two properly embedded surfaces $F$ and $G$ in $M$ are said to be parallel if there is an embedding $f: F \times I \rightarrow M$ such that $f((\operatorname{Bd} F) \times I) \subset \mathrm{Bd} M, f(F \times\{0\})=F$, and $f(F \times\{1\})=G$. The surfaces are said to be parallel/Bd (read "parallel modulo boundary") if $\mathrm{Bd} F=\mathrm{Bd} G$ and there is an embedding $f: F \times I \rightarrow M$ such that $f(F \times\{0\})=F$ and $f(\mathrm{Cl}(\mathrm{Bd}(F \times I)-(F \times\{0\})))=G$. A surface $S \subset M$ is said to be boundary parallel if $S$ is parallel/Bd to a surface in $\mathrm{Bd} M$. Analogous definitions apply to spanning arcs and simple closed curves embedded in a surface.

The reader is referred to [4], [8], [9], and [11] for discussions of the terms irreducible, incompressible, boundary irreducible, and boundary incompressible.

Consider $S \times I$, where $S$ is a surface. If $S \times\{0\}$ is identified with $S \times\{1\}$ by means of a homeomorphism which reverses the orientations induced from $S \times I$, the result is a manifold. If the manifold $M$ may be constructed in such a manner, we say that $M$ is fibered over $S^{1}$. The surface $S$ is called the fiber.

A knot is the image of a tame embedding of $S^{1}$ in $S^{3}$. Two knots are equivalent, or of the same knot type, if there is a homeomorphism of $S^{3}$ which takes one to the other. A knot space is the closure of the complement of a regular neighborhood of a knot in $S^{3}$. Equivalent knots have homeomorphic knot spaces. The group of a $k n o t$ is the fundamental group of its knot space. A Neuwirth knot is one whose knot space is fibered over $S^{1}$. These knots have been investigated in [6].

Let $M$ be a manifold with $\mathrm{Bd} M \neq \varnothing$. Suppose $m \subset \mathrm{Bd} M$ is an orientable simple closed curve. Let $A$ be a regular neighborhood of $m$ in $\mathrm{Bd} M$. Thus $A$ is an annulus in $\mathrm{Bd} M$. If the 3-cell $D^{2} \times I$ is attached to $M$ by identifying ( $\left.\mathrm{Bd} D^{2}\right) \times I$ with $A$, the result is a new manifold. Such a process is called sewing a pillbox along $m$.

A simple closed curve $m$ in the boundary of a knot space is called a meridian if sewing a pillbox along $m$ yields a 3-cell. Every knot space has a meridian, but it is unknown whether any two meridians are isotopic in the boundary of the knot space.

Two homeomorphisms $f, g: X \rightarrow Y$ are said to be isotopic if there is a map $H: X \times I \rightarrow Y$ such that $h_{\tau}=\left.H\right|_{X \times\{\tau\}}$ is a homeomorphism onto $Y, h_{0}=f$, and $h_{1}=g$. Two subspaces $Z_{1}$ and $Z_{2}$ of $X$ are isotopic if there is a homeomorphism $h: X \rightarrow X$ which is isotopic to the identity map and which has the property that $h\left(Z_{1}\right)=Z_{2}$. The act of replacing a subspace by one which is isotopic to it is called a deformation.

The surface $S \subset S^{3}$ is said to span the knot $K \subset S^{3}$ if $\operatorname{Bd} S=K$. If the surface $S$ 
spans the knot $K$, then $S$ is said to be an incompressible spanning surface if $S \cap \mathrm{Cl}\left(S^{3}-N(K)\right)$ is incompressible in $\mathrm{Cl}\left(S^{3}-N(K)\right)$. (Here $N(K)$ is assumed to be sufficiently small with respect to $S$.) It is known [2] that any two incompressible spanning surfaces for a Neuwirth knot $K$ are isotopic in the knot space determined by $K$.

A system of surfaces in $M$ is a collection of pairwise disjoint surfaces. Two systems of surfaces in a manifold are said to be in general position if their intersection consists entirely of spanning arcs and simple closed curves at which the surfaces meet transversely. It is known [5] that any two systems of surfaces may be placed in general position by performing a deformation in an arbitrarily small neighborhood of either system.

The following technical lemma will be used several times. Its proof is straightforward.

LEMMA 1. If $S_{1} \subset M_{1}$ and $S_{2} \subset M_{2}$ are surfaces other than disks which are incompressible and boundary incompressible with respect to the components $B_{1}$ of $\mathrm{Bd} M_{1}$ and $B_{2}$ of $\mathrm{Bd} M_{2}$, respectively, and if $M_{1}$ is attached to $M_{2}$ by identifying an incompressible surface $A_{1} \subset B_{1}$ with an incompressible surface $A_{2} \subset B_{2}$ in such a manner that $S_{1} \cap A_{1}$ is identified with $S_{2} \cap A_{2}$, then the resulting surface $S=S_{1} \cup S_{2}$ is incompressible in the new manifold $M$.

3. Closed incompressible surfaces in knot spaces. Let $T \subset S^{3}$ be an unknotted torus which determines a genus one Heegard Splitting $(V, W)$ of $S^{3}$. Since $V$ may be embedded in Euclidean 3-space, we may assume that $V$ inherits the cylindrical coordinatization $V=\left\{(r, \theta, z) \mid(r-4)^{2}+z^{2} \leqq 4\right\}$. Thus

$$
D_{1}=\left\{(r, \theta, z) \mid(r-4)^{2}+z^{2} \leqq 4, \theta=0\right\}
$$

is a meridian disk in $V$, and the curve $\{(2, \theta, 0)\} \subset \mathrm{Bd} V$ bounds a meridian disk $D_{2}$ in $W$.

The torus $T^{\prime}=\left\{(r, \theta, z) \mid(r-4)^{2}+z^{2}=1\right\} \subset V$ is parallel to $T$ and determines the Heegard Splitting $\left(V^{\prime}, W^{\prime}\right)$ of $S^{3}$, with $V^{\prime} \subset V$ and $W \subset W^{\prime}$. Let $D_{1}^{\prime}=D_{1} \cap V^{\prime}$ and $D_{2}^{\prime}=D_{2} \cup\{(r, \theta, 0) \mid 2 \leqq r \leqq 3\}$, so that $D_{1}^{\prime}$ and $D_{2}^{\prime}$ are meridian disks in $V^{\prime}$ and $W^{\prime}$, respectively. Define a knot $K \subset T^{\prime}$ by

$$
K=\{(r, \theta, z) \mid r=4+\cos \theta / 2,0 \leqq \theta \leqq 4 \pi, z=\sin \theta / 2\} .
$$

Note that $K$ is the torus knot $K_{(2,1)}$, which is trivial [3, p. 92].

The observation that $K$ meets each fiber of the product $V=D_{1} \times S^{1}$ exactly twice makes it apparent that $M=\mathrm{Cl}(V-N(K))$ inherits, from $V$, a fibering over $S^{1}$. The fiber is a planar surface with three boundary components. Let $R=\mathrm{Bd} N(K)$; then Bd $M=R \cup T$.

LEMMA 2. The manifold $M$ is boundary irreducible.

Proof. Note that $M$ is fibered in such a way that it has a double cover which is the product of $S^{1}$ and a planar surface having three boundary components. But any such product is boundary irreducible, so $M$ is also boundary irreducible. 
Let $D_{2}^{\prime \prime} \subset W^{\prime}$ be a meridian disk chosen so that $D_{2}^{\prime \prime} \cap V=\{(r, \theta, 0) \mid 5 \leqq r \leqq 6\}$. Let $X$ be an arbitrarily small 3-cell neighborhood of the point $(3,0,0)$ in $V^{\prime}$, so that $X \cap T^{\prime}$ is a 2-cell in Bd $X$. Note that $X \cap D_{1}^{\prime}$ is a 2-cell which meets both $K$ and $\mathrm{Bd} D_{2}^{\prime}$ in precisely the point $(3,0,0)$. Replace $X \cap D_{1}^{\prime}$ by another 2-cell in $X$ which agrees with $\mathrm{Bd}\left(X \cap D_{1}^{\prime}\right)$ on $\mathrm{Cl}\left(\mathrm{Bd} X \cap\right.$ Int $\left.D_{1}^{\prime}\right)$, which meets $K$ only in the point $(3,0,0)$, and which meets $\mathrm{Bd} D_{2}^{\prime}$ in an arc. This new 2-cell may be obtained by a deformation of $X \cap D_{1}^{\prime}$ in $X$. If we do a similar deformation in a neighborhood of $(5,0,0)$ in $V^{\prime}$, we obtain a meridian disk $D_{1}^{\prime \prime} \subset V^{\prime}$ which meets $K$ in the points $(3,0,0)$ and $(5,0,0)$, which meets $\mathrm{Bd} D_{2}^{\prime}$ in an arc, and which meets $\mathrm{Bd} D_{2}^{\prime \prime}$ in an arc.

It follows that $D^{\prime}=D_{2}^{\prime} \cup D_{1}^{\prime \prime} \cup D_{2}^{\prime \prime}$ is a disk with $\mathrm{Bd} D^{\prime} \subset T^{\prime}$ and $\mathrm{Bd} D^{\prime} \cap K=\varnothing$. But neither $K$ nor Bd $D^{\prime}$ are trivial on $T^{\prime}$, so they must be parallel on $T^{\prime}$. Hence we may alter $D^{\prime}$ by a deformation in an arbitrarily small neighborhood of $T^{\prime}$ to obtain a disk $D$ with $\operatorname{Bd} D=K$. Without loss of generality we may assume that $N(K)$ is small with respect to $D$, so that $N(K) \cap D$ is an annulus. Let $K^{\prime}=D \cap R$ $=D \cap \operatorname{Bd} N(K)$ and let $S=M \cap D$. Thus $R \cap S=K^{\prime}$. The proof of the following lemma is immediate from the construction.

LEMMA 3. (a) $S$ is orientable.

(b) $S$ is connected.

(c) $\chi(S)=-1$.

(d) $S \cap T$ consists of two simple closed curves which are isotopic, on $T$, to $\mathrm{Bd} D_{2}$.

LEMMA 4. $S$ is incompressible in $M$.

Proof. Because $S$ is a subset of $D, S$ must be planar. If $S$ were compressible in $M$, there would exist a nonsingular disk $E \subset$ Int $M$ such that $E \cap S=\mathrm{Bd} E$, and Bd $E$ would not bound a disk in $S$. However, Bd $E$ would have to bound a disk $F \subset D$, so if we replaced $F$ by $E$, we would obtain a new disk $D^{\prime}$ in $S^{3}$ with $\operatorname{Bd} D^{\prime}=K$. This process would necessarily reduce the number of intersections with $T$, so $D^{\prime} \cap T$ would either be empty or would consist of a simple closed curve parallel, on $T$, to Bd $D_{2}$. The first case is impossible because $K$ is not homotopically trivial in $V$. The second case would imply that $K$ is homotopic to a generator of $\pi_{1}(V)$, another contradiction. Hence, $S$ is incompressible.

LEMMA 5. $S$ is boundary incompressible in $M$.

Proof. Assume $S$ is boundary compressible in $M$. Thus there is a nonsingular disk $E \subset M$ such that $E \cap S=\alpha, E \cap \mathrm{Bd} M=\beta, \mathrm{Bd} E=\alpha \cup \beta, \alpha$ and $\beta$ are arcs, $\alpha \cap \beta=\mathrm{Bd} \alpha=\operatorname{Bd} \beta$, and $\alpha$ is not boundary parallel in $S$. We shall contradict the existence of the disk $E$. There are two possible cases.

Case 1. Bd $\alpha$ is contained in one component $b$ of $\mathrm{Bd} S$. Because $S$ is orientable, and hence 2-sided, $\beta$ must meet only one side of $b$. But $\mathrm{Bd} M$ consists of tori, so $\beta$ must be parallel/Bd in $\mathrm{Bd} M$ to an arc $\alpha^{\prime} \subset b$. Thus there is a nonsingular disk $C \subset \mathrm{Bd} M$ with $\mathrm{Bd} C=\alpha^{\prime} \cup \beta$. But $C \cup E$ is a nonsingular disk with its boundary 
in $S$, and it may be moved slightly to lie in Int $M$. Because $S$ is incompressible, this says that $\alpha$ must be parallel/Bd, in $S$, to $\alpha^{\prime}$, which is a contradiction. Hence Case 1 cannot occur.

Case 2. Bd $\alpha$ meets two distinct components $b$ and $c$ of $\mathrm{Bd} S$. In this case $b$ and $c$ must be parallel curves on $T$, so they separate $T$ into two annuli. Let $A$ denote the annulus containing $\beta$. The surface $S^{\prime}=S \cup A$ must be orientable, because $S$ was 2-sided and the disk $E$ assures us that we did not destroy orientability in adding $A$. But the existence of $S^{\prime}$ in $V$ implies that $K^{\prime}$ is homologous to zero in $V$, a contradiction. Hence, Case 2 cannot occur. This exhausts all possibilities.

Let $Q$ be the knot space of a nontrivial Neuwirth knot. Among other things, this means that $Q$ is a compact manifold with torus boundary, that $\pi_{1}(Q) \neq Z$, and that $Q$ is fibered over $S^{1}$ so that each fiber is a surface with one boundary component. We also have a meridian $m \subset \mathrm{Bd} Q$ which meets each fiber of $Q$ exactly once.

Attach $Q$ to $\mathrm{Cl}\left(S^{3}-N(K)\right)$ by identifying Bd $Q$ with $R=\mathrm{Bd} N(K)$ in such a way that $m$ is identified with $K^{\prime}$ and the boundary of each fiber of $Q$ is identified with a boundary component of a fiber of $M$. Call the resulting closed manifold $G$.

LEMMA 6. $G$ is homeomorphic to $S^{3}$.

Proof. Let $D^{\prime}=D \cap \mathrm{Cl}\left(S^{3}-N(K)\right)$. If $I\left(D^{\prime}\right)$ is a product neighborhood of $D^{\prime}$ in $\mathrm{Cl}\left(S^{3}-N(K)\right)$, then $\mathrm{Cl}\left(S^{3}-\left(N(K) \cup I\left(D^{\prime}\right)\right)\right)$ is a 3-cell. But $Q \cup I\left(D^{\prime}\right)$ is also a 3-cell, and $G$ is obtained by identifying these 3-cells along their boundaries. Hence $G$ is homeomorphic to $S^{3}$.

Lemma 6 essentially says that we now have another embedding of the solid torus $\mathrm{Cl}\left(S^{3}-N(K)\right)$ in $S^{3}$. From this point on, we will be concerned with this new embedding, unless we make explicit mention to the contrary. In this new embedding, $W$ may be interpreted as the regular neighborhood of a simple closed curve. Hence $P=\mathrm{Cl}(G-W)$ is a knot space. Note that $P=Q \cup M$ with the appropriate identifications.

Lemma 7. $P$ is the knot of a nontrivial, prime, Neuwirth knot, and $\mathrm{Bd} D_{2}$ is a meridian for $P$.

Proof. The construction assures us that $P$ is fibered over $S^{\mathbf{1}}$ and that each fiber has one boundary component. If we attach a pillbox to $P$ along $\mathrm{Bd} D_{2}$, we obtain $G$ minus a 3-cell, which is a 3-cell. Hence $\mathrm{Bd} D_{2}$ is a meridian and $P$ is the knot space of a Neuwirth knot. Both $Q$ and $M$ are boundary irreducible, so Van Kampen's theorem assures us that $\pi_{1}(P) \neq Z$, and the knot is nontrivial.

It remains to be shown that $P$ is the knot space of a prime knot. Assume the contrary. Thus there exists a separating, nonsingular, incompressible annulus $A$ properly embedded in $P$. Each component of $\mathrm{Bd} A$ is homologous, in $\mathrm{Bd} P$, to $\mathrm{Bd} D_{2}$, and $A$ is not boundary parallel. Put $A$ in general position with respect to $R$, so that the intersection consists entirely of simple closed curves. Assume a minimal number of these curves. If any of these curves were trivial on $A$, it would 
also have to be trivial on the incompressible surface $R$, and it could be removed. Hence $A \cap R$ must consist entirely of boundary parallel simple closed curves on $A$. Let $\sigma$ be an outermost such simple closed curve on $A$, so that $\sigma$ and a boundary component $\tau$ of $A$ cobound an annulus in $M$. But this says that in the original embedding, some multiple of the curve $K$ was homologous to $\mathrm{Bd} D_{2}$, a contradiction. Thus $A \cap R=\varnothing$ and $A \subset M$.

Since $A$ separates $P, A$ must also separate $M$. Put $A$ in general position with respect to one fiber $F$ of $M$. Because each component of $\mathrm{Bd} A$ is homologous, in $T$, to $\mathrm{Bd} D_{2}$, we may assume that $A \cap F$ consists of simple closed curves and one arc $\alpha$. But $\alpha$ must connect the boundary components of $A$, so the simple closed curves may be eliminated. The fact that $A$ separated $M$ assures us that $\alpha$ is boundary parallel in $F$. Thus $A$ is boundary compressible in $M$, and hence boundary parallel in $M$, a contradiction. Thus the knot is prime.

Now construct $P$ as above with the additional qualification that $Q$ is the knot space of a composite Neuwirth knot. This means that there is a separating, incompressible, and boundary incompressible annulus $A$ properly embedded in $Q$. Each component of $\mathrm{Bd} A$ is isotopic, in $R$, to $K^{\prime}$.

Let $B \subset \mathrm{Bd} P=T$ be an annulus chosen so that $S \cap T \subset$ Int $B$ and $B$ inherits a product structure $I \times S^{1}$ from the fibering of $P$. Let $P_{1}$ and $P_{2}$ be two copies of $P$, and let subscripts identify the corresponding subspaces, e.g., $S_{1}, Q_{2}, A_{2}$, etc. Attach $P_{1}$ to $P_{2}$ by identifying $B_{1}$ with $B_{2}$, the identification map being chosen so as to reverse orientation, match the product structure, and identify $S_{1} \cap B_{1}$ with $S_{2} \cap B_{2}$. Call the resulting manifold $L$. Let $Z^{\prime}=S_{1} \cup S_{2}$, let $B=B_{1}=B_{2}$, and let $L^{\prime}=\mathrm{Cl}\left(L-\left(Q_{1} \cup Q_{2}\right)\right)$.

Lemma 8. The surface $Z^{\prime}$ is incompressible and boundary incompressible in $L^{\prime}$, and $\chi\left(Z^{\prime}\right)=-2$.

Proof. The first two statements follow immediately from Lemma 1, Lemma 4, and Lemma 5. The last statement follows from the observation that $\chi\left(Z^{\prime}\right)$ $=\chi\left(S_{1}\right)+\chi\left(S_{2}\right)=(-1)+(-1)=-2$.

Lemma 9. The manifold $L$ is the knot space of a composite Neuwirth knot, and either component of $\mathrm{Bd} B$ is a meridian.

Proof. Attach a pillbox $Y$ to $L$ along a component $\sigma$ of $\mathrm{Bd} B$, so that $Y \cap B=\sigma$. Note that $\sigma$ must bound a disk $E$ in $Y$. The disk $E$ separates $Y$ into two 3-cells $Y_{1}$ and $Y_{2}$, with $Y_{1} \cup Y_{2}=Y, Y_{1} \cap Y_{2}=E, Y_{1} \cap L \subset \operatorname{Bd} P_{1}$, and $Y_{2} \cap L \subset \operatorname{Bd} P_{2}$. But $P_{1} \cup Y_{1}$ and $P_{2} \cup Y_{2}$ are just 3-cells, and $L \cup Y$ is obtained by identifying them along the disk $B \cup E$ which is common to their boundaries. Hence $L$ is a knot space with meridian $\sigma$. However, the fibering over $S^{1}$ was preserved in the identification of $B_{1}$ with $B_{2}$, so $L$ is also fibered over $S^{1}$. The annulus $B$ assures us that the knot is composite. 
We now turn our attention to the task of constructing closed incompressible surfaces of arbitrarily high genus in $L$. Let $I\left(Z^{\prime}\right)$ be a product neighborhood of $Z^{\prime}$ in $L^{\prime}$. Identify $I\left(Z^{\prime}\right)$ with $Z^{\prime} \times I$, and let $n$ be a fixed positive integer. Define $Z_{1}^{\prime}=Z^{\prime} \times\{i / 2 n\}$ for $0 \leqq i<2 n$. Similarly define $A_{j k}=A_{j} \times\{k / n\}$ in $I\left(A_{j}\right) \subset Q_{j}$ for $j=1,2$, and $0 \leqq k<n$. The sets $\left\{\left(\mathrm{Bd} Z_{i}^{\prime}\right) \cap Q_{j}\right\}$ and $\left\{\mathrm{Bd} A_{j k}\right\}$ consist entirely of simple closed curves on $\mathrm{Bd} Q_{j}$, all of which are isotopic, on $\mathrm{Bd} Q_{j}$, to meridians.

We may assume that the $A_{1 k}$ 's have been isotoped, in $Q_{1}$, so that one boundary component of $A_{1 k}$ is identified with $Z_{n+k}^{\prime} \cap Q_{1}$ and the other is identified with $Z_{n-k-1}^{\prime} \cap Q_{1}$ for $0 \leqq k<n$. Similarly we may assume that $A_{2 k}$ has one boundary component identified with $Z_{n+k-1}^{\prime} \cap Q_{2}$ and the other with $Z_{n-k-2}^{\prime} \cap Q_{2}$ for $0 \leqq k<n$. (All subscripts are taken modulo $2 n$.) The result is a connected closed surface $Z$. Figure 1 is a schematic diagram of $Z$.

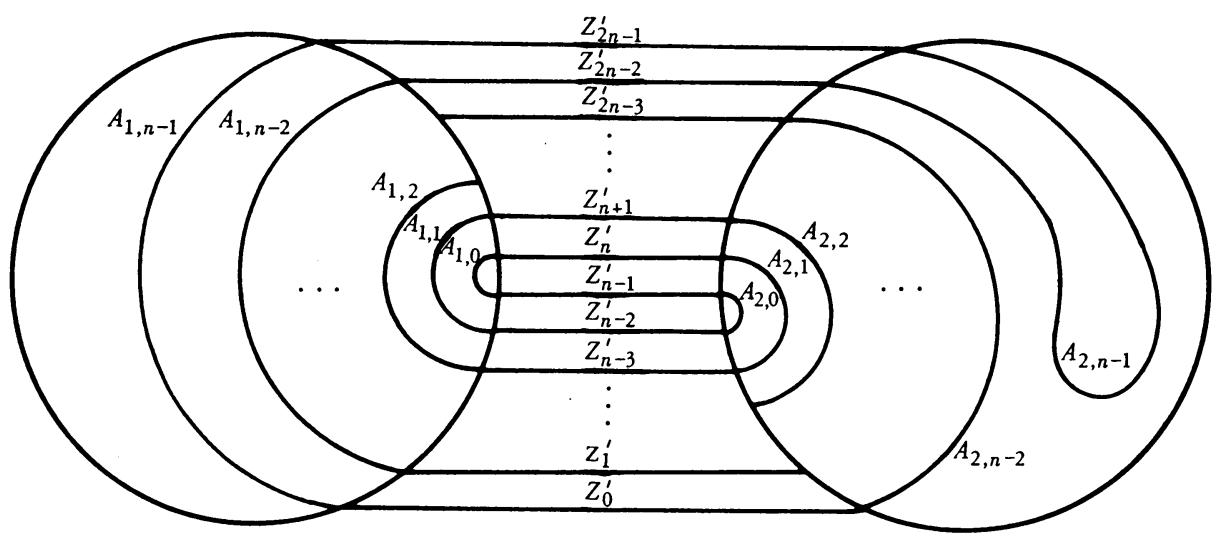

Figure 1

Lemma 10. The surface $Z$ is orientable, it separates $L$, and it has genus $2 n+1$.

Proof. Since $Z$ is closed and connected, and $Z \subset L \subset S^{3}, Z$ must be orientable and must separate $L$. By the construction, $\chi(Z)=2 n(-2)=-4 n$, so the genus of $Z$ is $2 n+1$.

LeMma 11. The surface $Z$ is incompressible in $L$.

Proof. Assume the contrary. Thus there exists a nonsingular disk $D \subset$ Int $L$ such that $D \cap Z=\operatorname{Bd} D$ and $B d D$ does not bound a disk in $Z$. Let $L^{\prime \prime}$ be the closure of the component of $L-Z$ which contains Int $D$. Every component of ( $\left.L^{\prime \prime} \cap \operatorname{Bd} L^{\prime}\right)$ $-\mathrm{Bd} L$ is a separating annulus which is necessarily incompressible by Lemma 2 and the fact that $Q_{j}$ is boundary irreducible for $j=1,2$. Put $D$ in general position with respect to $L^{\prime \prime} \cap \mathrm{Bd} L^{\prime}$. Since the latter is incompressible, we may assume that all intersections consisting of simple closed curves have been removed. Hence the intersection consists entirely of spanning arcs on $D$. Assume a minimal number of such spanning arcs, and let $\alpha$ denote an outermost one on $D$. Let $\beta \subset \operatorname{Bd} D$ be an arc such that $\beta \cap \operatorname{Bd} L^{\prime}=\operatorname{Bd} \alpha=\operatorname{Bd} \beta$. Thus $\beta$ is either in some $Z_{i}^{\prime}$ or in some $A_{j k}$. 
However, $Z_{i}^{\prime}$ is boundary incompressible in $L^{\prime}$ and $A_{j k}$ is boundary incompressible in $Q_{j}$ for $j=1,2$, and $0 \leqq k<n$. Thus $\alpha$ can be eliminated by an isotopy of $D$, and we may assume that $D \cap \mathrm{Bd} L^{\prime}=\varnothing$. But now $\mathrm{Bd} D$ must be entirely contained in some $Z_{i}^{\prime}$ or $A_{j k}$. Since $Z_{i}^{\prime}$ is incompressible in $L^{\prime}$ and $A_{j k}$ is incompressible in $Q_{j}$, we have arrived at a contradiction. Thus, $Z$ is incompressible.

We have constructed a composite Neuwirth knot whose complement $L$ contains closed incompressible surfaces of arbitrarily high genus. However, if we let our $L$ play the role of $Q$ in the construction immediately preceding the statement of Lemma 7, we will have constructed the knot space of a prime Neuwirth knot with the above property. Also, this procedure may be repeated indefinitely, and at each stage we get the knot space of a prime Neuwirth knot whose genus is twice the genus of the knot of the preceding knot space. Since genus is an invariant of knot type, we have proved the following theorem.

THEOREM 1. There exist infinitely many distinct, prime, Neuwirth knots, each of which has the property that its complement contains closed, incompressible surfaces of arbitrarily high genus.

4. A knot with infinitely many incompressible spanning surfaces. Consider the manifold $L$ of Lemma 9 along with the annulus $B \subset L$. Note that $B$ is boundary incompressible in $L$. Let $\sigma$ be an outermost (on $B$ ) simple closed curve component of $B \cap Z$, so that $\sigma$ and a component $\tau$ of $\mathrm{Bd} B$ cobound an annulus $B^{\prime} \subset B$. Cut $Z$ along $\sigma$ and attach to each boundary component of $\mathrm{Cl}(Z-(N(\sigma) \cap Z))$ an annulus parallel to $B^{\prime}$. The result is a properly embedded surface $Z^{\prime \prime} \subset L$, and each component of $Z^{\prime \prime} \cap \operatorname{Bd} L=\mathrm{Bd} Z^{\prime \prime}$ is a meridian.

LEMMA 12. The surface $Z^{\prime \prime}$ is connected.

Proof. Assume $\sigma \subset Z_{i}^{\prime}$. By the construction, $\sigma$ does not separate $Z_{i}^{\prime}$. Hence $\sigma$ cannot separate $Z$, and $Z^{\prime \prime}$ is connected.

Lemma 13. The surface $Z$ is incompressible in $L$.

Proof. Assume the contrary. Let $D \subset \operatorname{Int} L$ be a nonsingular disk so that $D \cap Z^{\prime \prime}=\operatorname{Bd} D$ and Bd $D$ does not bound a disk in $Z^{\prime \prime}$. The disk $D$ may be moved by an isotopy, which keeps $\operatorname{Bd} D$ in $Z^{\prime \prime}$, until $D \cap Z=\operatorname{Bd} D$. This, however, contradicts the incompressibility of $Z$. Hence $Z^{\prime \prime}$ is incompressible.

LEMMA 14. The surface $Z^{\prime \prime}$ is boundary incompressible in $L$.

Proof. Assume that there exists a nonsingular disk $D \subset L$ such that $\operatorname{Bd} D$ $=(D \cap \operatorname{Bd} L) \cup\left(D \cap Z^{\prime \prime}\right), D \cap \operatorname{Bd} L$ is an $\operatorname{arc}$, and $\mathrm{Bd}(D \cap \operatorname{Bd} L)=\mathrm{Bd}\left(D \cap Z^{\prime \prime}\right)$. Put $D$ in general position with respect to $B \cup \operatorname{Bd} Q_{1} \cup \operatorname{Bd} Q_{2}$ and assume that the number of components in this intersection is minimal. Since $B, \operatorname{Bd} Q_{1}$, and $\operatorname{Bd} Q_{2}$ are all incompressible in $L$, there can be no simple closed curves in the intersection. Exactly the same argument that was used in the proof of Lemma 11 assures us that 
$D \cap\left(\operatorname{Bd} Q_{1} \cup \operatorname{Bd} Q_{2}\right)=\varnothing$. Let $\alpha$ be an outermost $\operatorname{arc}($ on $D)$ in $D \cap B$. If $\alpha \cap \tau=\varnothing$, let $D^{\prime} \subset D$ be a disk chosen so that $\mathrm{Bd} D^{\prime} \subset(\alpha \cup \operatorname{Bd} D)$ and $D^{\prime} \cap B=\alpha$. Assume $D^{\prime} \subset M_{j}, j=1$ or 2 . This implies that $\mathrm{Cl}\left(\mathrm{Bd} D^{\prime}-\alpha\right)$ must be contained in a copy of $S_{j}$, and Lemma 5 assures us that $\alpha$ could have been eliminated. A similar argument applies if $\alpha \cap \tau \neq \varnothing$.

Now consider Zieschang's projection of the knot $9_{46}$ [12], as shown here in Figure 2. This knot is nontrivial and has the genus one spanning surface $S$ as indicated. It follows that $S$ must be incompressible.

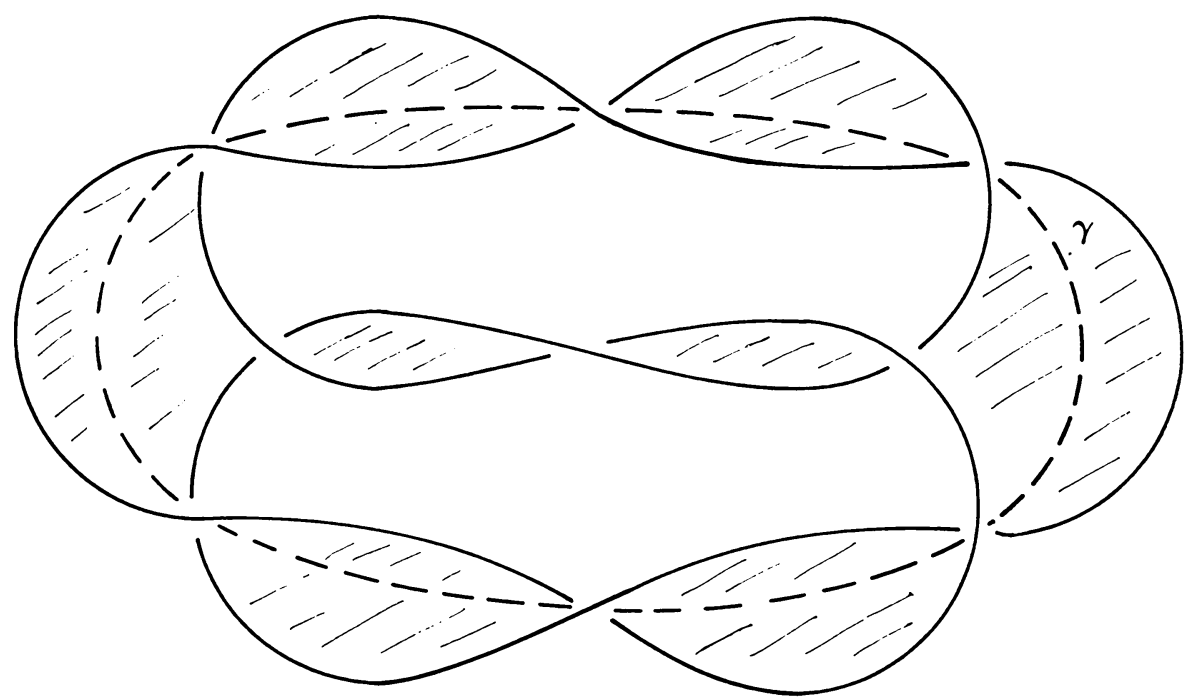

FIGURE 2

Let $C=S \cap N(\gamma)$ be an annular regular neighborhood of the nontrivial curve $\gamma$ in $S$. Since $\gamma$ has been chosen so that $C$ is unknotted and untwisted in $S^{3}, C$ may be assumed to be embedded in a 2-sphere in $S^{3}$. It follows that there is a nonsingular disk $D \subset S^{3}$ such that $D \cap C=\gamma=\mathrm{Bd} D$. Thus $D^{\prime}=D \cap \mathrm{Cl}\left(S^{\mathbf{9}}-N(\gamma)\right)$ is a meridian disk in the solid torus $\mathrm{Cl}\left(S^{3}-N(\gamma)\right)$. The incompressibility of $S \cap \mathrm{Cl}\left(S^{3}-N(K)\right)$ in $\mathrm{Cl}\left(S^{3}-N(K)\right)$ assures us that $S \cap \mathrm{Cl}\left(S^{3}-(N(K) \cup N(\gamma))\right)$ is boundary incompressible with respect to $\mathrm{Bd} N(\gamma)$.

Re-embed the solid torus $\mathrm{Cl}\left(S^{3}-N(\gamma)\right)$ in $S^{3}$ by identifying Bd $N(\gamma)$ with Bd $L$ in such a way that $\mathrm{Bd} D^{\prime}$ is identified with a meridian on $\mathrm{Bd} L$. The curve $K$ now represents a different knot in $S^{3}$. If $S \cap \mathrm{Bd} N(\gamma)$ is identified with $\mathrm{Bd} Z^{\prime \prime}$, the result is an orientable surface of genus $2 n+1$ which spans this new knot. Lemmas 1,13 , and 14 , together with the boundary incompressibility of

$$
S \cap \mathrm{Cl}\left(S^{3}-(N(K) \cup N(\gamma))\right)
$$

in $\mathrm{Cl}\left(S^{3}-(N(K) \cup N(\gamma))\right)$ with respect to $\mathrm{Bd} N(\gamma)$ assure us that this surface is incompressible. Hence, this new knot is nontrivial. However, if $S \cap \mathrm{Bd} N(\gamma)$ is identified with $\mathrm{Bd} B$, the result is a spanning surface of genus one. We have proved 
THEOREM 2. There exists a genus one knot which has incompressible spanning surfaces of arbitrarily high genus.

\section{BIBLIOGRAPHY}

1. W. R. Alford, Complements of minimal spanning surfaces of knots are not unique, Ann. of Math. (2) 91 (1970), 419-424.

2. Gerhard Burde and Heiner Zieschang, Neuwirthsche Knoten und Flächenabbildungen, Abh. Math. Sem. Univ. Hamburg 31 (1967), 239-246. MR 37 \#4803.

3. R. H. Crowell and R. H. Fox, Introduction to knot theory, Ginn, Boston, Mass., 1963. MR 26 \#4348.

4. Wolfgang Haken, Some results on surfaces in 3-manifolds, Studies in Modern Topology, Math. Assoc. Amer.; distributed by Prentice-Hall, Englewood Cliffs, N. J., 1968, pp. 39-98. MR 36 \#7118.

5. John Hempel, 3-manifolds, Course Notes for Math. 550, Rice University, Houston, Texas.

6. L. Neuwirth, Knot groups, Ann. of Math. Studies, no. 56, Princeton Univ. Press, Princeton, N. J., 1965. MR 31 \#734.

7. C. B. Schaufele, The commutator group of a doubled knot, Duke Math. J. 34 (1967), 677-681. MR 35 \#7328.

8. F. Waldhausen, Gruppen mit Zentrum und 3-dimensionale Mannifaltigkeiten, Topology 6 (1967), 505-517. MR 38 \#5223.

9. - Eine Klasse von 3-dimensionalen Mannifaltigkeiten. I, Invent. Math. 3 (1967), 308-333. MR 38 \#3880.

10. —_, Eine Klasse von 3-dimensionalen Mannifaltigkeiten. II, Invent. Math. 4 (1967), 87-117. MR 38 \#3880.

11. - On irreducible 3-manifolds which are sufficiently large, Ann. of Math. (2) 87 (1968), 56-88. MR 36 \#7146.

12. H. Zieschang, On a problem of Neuwirth on knot groups, Dokl. Akad. Nauk SSSR 153 (1963), 1017-1019= Soviet Math. Dokl. 4 (1963), 1781-1783. MR 31 \#4025.

\section{University of Michigan, Flint College,}

Flint, Michigan 48503 